\title{
SAGA GIS-A REVIEW
}

\begin{tabular}{|l|l|}
\hline Ravikiran M.D.T. & Badari Nath. K \\
II yr. M. Tech., Dept. of CSE, & Asst. Prof., Dept. of CSE, \\
R.V.C.E. & R.V.C.E \\
Bangalore, India & Bangalore, India \\
ravikirandevadiga@ gmail.com & badarinath.kb@ rvce.edu.in \\
\hline
\end{tabular}

\begin{abstract}
This paper explores various aspects of the SAGA GIS software right from its architecture to the way it can be put into use. The existing projects along with the other GIS tools that are similar in context are the advancements in this area. A review of the internal components of SAGA GIS and it's the way in which it can be put to use is described hereby.
\end{abstract}

Index Terms-HPC, SAGA GIS, GRASS, QGIS, Open source software.

\section{INTRODUCTION}

"The application of GIS is limited only by the imagination of those who use it" - Jack Dangermond.

Geographic Information System (GIS) is an organized computer based information system for the analysis of geographic features [1]. It is designed to build, manage, evaluate, and show all types of geographically or spatially referenced data. The information in a GIS describes entities that have a physical location and extent in some spatial region of interest, while queries involve identifying these entities based on their spatial and temporal attributes and relationships between entities [10]. Geographic data refers to spatial data in terms of their position with respect to a known co-ordinate system, their attributes (which are unrelated to their position) and their spatial inter-relationships with one another. Integration of imagery with vector data is now a necessity for a full-featured GIS system. Imagery was once thought to be the exclusive domain of image processing systems, but is now often required as a backdrop for vector, or other data, types. High performance computers [9] are formed as a result of aggregation or clustering of several computers. A computer chosen at random from any cluster would have one to four processors each of which is made up of two to four cores. The purpose of which is to gain higher performance than the one that would have otherwise been obtained from a sole desktop computer or a workstation. It helps to tackle the process of solving complex problems from fields such as engineering, business, science, etc. System for Automated Geo-scientific Analyses (SAGA)[2] is open source hybrid GIS software with a special "Application Programming Interface" for geographic data processing. The SAGA GIS API supports grid data like digital terrain models and satellite images, vector data, and tables. It is designed to tackle real world scenarios and education sector. It is compatible with both Windows and Linux systems.

\section{LITERATURE SURVEY}

The paper [3] illustrates how geo-information science can improve the traditional agricultural practices by making use of High Performance Computing (HPC) which in turn uses supercomputers and computer clusters to solve complex problems like agricultural activity monitoring. It also provides an insight into some of the open source geo information applications which make use of HPC for a sustainable agriculture.

The paper [4] examines the use of image processing and remote sensing in land usage changes mapping for Palladam Taluk between 1972(Topographic sheets) and 2011(satellite images). Classification of satellite image was done with the help of SAGA GIS. The classified image was converted into vector format followed by an estimation of the total area of each class.

The paper [1] performs an assessment on the influence of changing land-use patterns on the groundwater quality in the Maheshwaram watershed, near Hyderabad, India. Zones of sustainable and unsustainable groundwater use were demarcated for better decision making related to municipal land allotment in this rapidly urbanizing region. The open source GIS package SAGA GIS 2.0.4 was employed for all the GIS tasks.

The paper [6] presents a geomorphological survey of the Olvera area (Cadiz province, Betic Ranges, Spain) by taking into account different landforms. An Open Source GIS, i.e. SAGA GIS was used for the survey with the key aim of having the project-derived data available over the Web.

The paper [7] illustrates methods to create more detailed raster soil maps in areas with sparse point data. Legacy conventional soil maps are often the main source of soil-landscape information. Each soil series training area was then used in a single-class maximum likelihood supervised classification in SAGA GIS.

The paper [8] describes the basic necessities of any error propagation methodology. Streams are extracted using the open source SAGA GIS via the RSAGA library. Efficiency of such a method can be improved by correlating the propagated uncertainty of the derived stream network with various land surface parameters.

\section{SAGA GIS ARCHITECTURE}

SAGA GIS was developed at the Department of Physical Geography, University of Gottingen, Germany with a purpose of editing spatial data by making use of spatial algorithms. It 


\section{Asia Pacific Journals}

is basically a GIS software that aids scientists with a platform that is easy to comprehend, in order to implement geoscientific methods with the help of API. Apart from API, SAGA comprises of modules, libraries and an easily visualizable GUI.

\section{A. Features of SAGA GIS}

- Written in $\mathrm{C}++$, thus possess an object oriented system design.

- Immense capability to handle geospatial data.

- Free and Open Source Software (FOSS)

- Cross platform compatibility i.e. Linux and Windows

- Easy to use GUI

- Scripting via command line, Python, Java, R

- Image analysis: filters, supervised classification, PCA, FFT.

- Geostatistics: GWR, variograms, ordinary \& universal Kriging.

- Terrain analysis: morphometry, hydrology, illumination, classification.

- Vector tools: clipping, buffer zones, raster to vector conversion.

- Portable software

- More than 450 freely available functions for geodata analysis

\section{B. System Architecture}

\begin{tabular}{|c|c|c|c|c|}
\hline \multicolumn{5}{|c|}{$\begin{array}{c}\text { GUI: Graphical User Interface } \\
\text { User interaction, visualization }\end{array}$} \\
\hline \multicolumn{3}{|c|}{ MODULE MANAGEMENT } & \multicolumn{2}{|c|}{ DATA MANAGEMENT } \\
\hline \multicolumn{5}{|c|}{ MODULE LIBRARIES } \\
\hline Climate - Tools & Grid & Imagery & Simulation & Terrain Analysis \\
\hline ImportExport & Projection & Shape: & Visualiza & Garden \\
\hline \multicolumn{5}{|c|}{ API: Application Program Interface } \\
\hline \multicolumn{5}{|c|}{ Classes, functions, scientific modules, object models } \\
\hline
\end{tabular}

Fig. 1: SAGA architecture

System architecture of SAGA GIS is modular as shown in Fig 1. Application Programming Interface (API) forms the base of SAGA GIS. It comprises of data object models, basic definitions for the programming of scientific modules and numerous helpful classes and functions that aid the implementation. Module libraries are containers for the scientific methods in form of so called modules. API as well as module libraries co-exist as Dynamic Link Libraries (DLL) and have to be accessed through a front end program. Two kinds of front end tools available in SAGA GIS: 1.Graphical User Interface (GUI) 2. Command line interpreter tool. In case of GUI, the control of the system lies with the user. It is also responsible for module and data management as well as for data visualizations. Similarly execution of modules can be done using the command line interpreter.

\section{Graphical User Interface}

As and when user executes specific modules from the GUI, he/she can manage and visualise data as well as perform data analyses and manipulations. Along with menu, tool and status bars, SAGA GIS consists of three additional control elements: workspace control, message control, properties control.

1) The workspace control: It has sub windows for the modules, data and maps workspaces. Each workspace shows a tree view, through which associated workspace objects can be accessed. Loaded module libraries are listed in the modules workspace together with a list of their modules. Similarly created map views will be listed in the maps workspace and data objects in the data workspace, hierarchically sorted by their data type.

2) The object properties control: Dependent on which object in a workspace is selected, the object properties control shows an object specific set of sub windows. In case a module is selected, the settings window is populated with the module parameters from where the user can choose data sets and other options for the module execution. The description window gives information about the module and its parameters, as supplied by the module programmer. In case a data object is selected the settings window gives control to data specific properties, like data set name, memory handling and display behaviour. Other object windows allow to edit the attributes of vector layers or display a legend for a selected map.

3) The message control: It contains three sub windows for general, module specific and error notifications.

Most modules create new data sets as result of their calculation, which will automatically be added to the data workspace, so that these can be saved, used as input for further calculations, or displayed in a map. Data visualization in the form of maps can be in $3 \mathrm{~d}$ format, histograms and scatter plots.

\section{Modules}

1) Data import and export: SAGA GIS provides several types of filters to common data formats, including various image and GPS formats. Most flexible is a raster data import tool that uses the Geospatial Data Abstraction Library (GDAL), which alone supports about 40 different file formats.

2) Cartographic projections and geo-referencing: Once data have been imported, the next necessary step is in most cases to geo-reference or to project it, so that all spatial data sets of a project belong to one single coordinate system. SAGA GIS provides two cartographic projection libraries, the Geographic Translator GeoTrans library and the Proj.4 library. Both libraries work for raster as well as for vector data and provide various projections for free definable cartographic parameters. 
3) Numerous raster and vector data tools: Many modules exist for manipulation and analysis of vector data, like merging of layers, selection of shapes, attribute table manipulation, type conversion and automated document creation. Standard operations on vector data are polygon layer intersections and vector data creation from raster data, e.g. creation of contour lines.

4) Image processing: A number of filter algorithms have been implemented for smoothing, sharpening or edge detection. Classifications can be performed using cluster analysis or a supervised procedure, e.g. minimum distance and maximum Likelihood classification.

5) Terrain analysis: Modules for the construction and preparation of raster data, allow the resampling, closing of gaps and the manipulation of value by user defined rules. A very flexible standard tool is the raster calculator, where a user defined formula is used to combine an arbitrary number of raster layers.

6) Geo-statistics: Statistical analyses for raster data include zonal statistics, residual and variance analysis. Single and multiple regression analyses relate point data to raster data and allow the extrapolation of values.

\section{E. API}

SAGA GIS has API's such as CSG Array, Buffer, Bytes, Class Statistics, Classifier Supervised, Colors, Data Collection, Grid System, Module, Radius, etc.

\section{PROJECTS IMPLEMENTED USING SAGA GIS}

1) CARBIOCIAL: Carbon sequestration, biodiversity and social structures in Southern Amazonia [12]: models and implementation of carbon-optimized land management strategies. Subproject 10 titled "Development and implementation of a hierarchical model chain for modelling regional climate variability and climate change over southern Amazonia". The main aim of which is to analyze and model present and future atmospheric processes, boundary layer dynamics and spatiotemporal climate variations in southern Amazonia under different climate and land use scenarios.

2) The Future Okavango: Scientific support for sustainable land and resource management in the Okavango basin [13]. Subproject 9.2 titled "GIS-based landscape analyses, environmental modelling, and decision support for integrated resource management". The purpose is to develop and implement an integrated geospatial data analysis and decision support system within a GIS environment, featuring landscape analyses, modelling applications and an integrated assessment and optimization of landscape and resource management strategies for alternative climate and land use scenarios. The applications will integrate and aggregate methods, results and information from different subprojects within SAGA GIS.
3) CHELSA: Climatologies at High Resolution for the Earth's Land Surface Areas [14]. This project tries to investigate broad-scale ecological and biogeographic patterns in order to document general trends in the spatial distribution of biodiversity. Thus it aims to get a clear picture of the processes leading to uneven geographic distribution and to provide knowledge, theory, and tools for applied questions in conversation and basic ecology.

4) LEM: Landscape evolution model [14]. This project tries to develop a model for the Spatial Prediction of Periglacial Deposits.

5) SAGA-REKLIM: Climate Change and Forestry [15]: Researches and developments for a SAGA GIS based problem oriented regionalization of spatially distributed climate data.

6) CliSAP: Integrated Climate System Analysis and Prediction [16]. Spatial high resolution regionalization of urban climates, integrating statistical-dynamical downscaling and remote sensing techniques. It aims for long range climate visions.

\section{COMPARATIVE STUdY}

Similar to SAGA GIS, several other GIS softwares do exist. Features of a few of them are discussed below.

\section{A. SAGA GIS vs. GRASS}

Two of the most widely used open source desktop GIS software for the analysis of DEM [11] (Digital elevation model) are SAGA (System for Automated Geoscientific Analyses) GIS and GRASS (Geographic Resources Analysis Support System) GIS. SAGA GIS is in development since 2001 aiming at simplifying the implementation of new algorithms for spatial data analysis. GRASS itself is a collection of modules. Although originally a Linux-based project, the most recent version of GRASS (6.3) is now also available for MS Windows machines.

The latest version of SAGA GIS (2.1.3.) contains 48 libraries with 300 modules; GRASS [17] 6.3 contains over 350 routines. Functionality provided by SAGA and GRASS for the analysis of DEMs is much more enhanced than proprietary low-end products such as the basic installation of ArcGIS 9.2. By linking SAGA GIS/GRASS with $R$ environment for statistical computing, a powerful combination is created that allows fusion of GIS and statistical functionality in the same code. The results of this set of comparisons shows that there are indeed some differences between the two software: SAGA GIS seems to be more computationally efficient (2-3 times faster), GRASS generates more accurate streams networks, and in general offers more sophistication considering analysis of elevation data. Rest of the functionality such as that of DEM generation, image processing, vector/raster conversion is comparable in both the softwares.

SAGA GIS has a user friendly approach in cases such as manipulation of maps, zooming into the data and controlling 


\section{Asia Pacific Journals}

the processing from external applications. GRASS, on the other hand is powerful as a tool for processing, but its interactive display characteristics are limited. SAGA GIS lacks help documents that explain different functions, how to set-up different parameters etc.

\section{B. SAGA GIS vs. QGIS}

GIS software that are widely used in teaching and research are SAGA GIS and QGIS ${ }^{[16]}$. QIS can also be applied to the field of cartography and geo-informatics. These software packages offer rich functionality for working with vector and raster data, as well as access to Web services, cartographic design of digital maps, process modelling using graphical modeller or Python language. Additionally, with the SEXTANTE9 modeller it is possible to combine the $\mathrm{R}$ programming language together with SAGA GIS and QGIS features, as well as the GRASS GIS10, GDAL/OGR11, Orfeo Toolbox12 functions, and others.

\section{IMPLEMENTATION}

SAGA GIS can be implemented by means of scripting languages such as Python, $\mathrm{R}$ etc. thus enabling the processing of multiple layers at one go.

\section{A. PYTHON}

Python has the capability to execute external programs. This can be done in a much better way by using Python script provided by GRASS GIS. It is called as grass.py. It does so by masking native Python complexity. The grass.py script consist of functions to invoke GRASS modules. The usage is similar to that of a call to any of the SAGA GIS modules.

To call a SAGA GIS module from a Python script simply import grass and use the following function call:

$$
\text { run_command("saga_cmd ....") }
$$

where the .... represent the arguments to the saga_cmd as would be used from the command line.

For instance, if terrain analysis for a number of grids from the GUI is to be done, pick one among the Terrain Analysis modules, be it Local Morphometry module, repeatedly, once for each grid. Using Python and grass.py on the other hand, you can process all the grids in batch, as shown here for Windows (assumes a directory called 'yourPath' full of grids only), but would be similar for Linux.

Python script enables access to the SAGA GIS API through a special interface called the SAGA GIS Python API. This interface is distributed with the SAGA GIS binaries and has to be compatible with the same Python interpreter as used by the scripts which use it.

\section{B. RSAGA}

SAGA GIS can be accessed from within the $\mathrm{R}$ statistical data analysis environment with the help of the RSAGA package [7]. It provides functions that make use of the command line interface of SAGA GIS, saga_cmd.exe, to execute SAGA GIS modules. The RSAGA [9] package consists of the following general components:

1. The RSAGA core provides low-level access to the SAGA GIS command line program through the rsaga.geoprocessor function, which converts $\mathrm{R}$ arguments into a command that is executed by the operating system. It also accesses SAGA GIS module libraries and help files to give information on available modules (function rsaga.get.modules) and command line options (rsaga.get.usage).

2. The RSAGA modules implements a series of interface functions that allow the $\mathrm{R}$ user to run SAGA GIS modules from within the accustomed $\mathrm{R}$ environment. For example: rsaga.local.morphometry and rsaga.flow.accumulation. These functions are often more easy to use than the corresponding SAGA modules; eg: flow accumulation algorithms can be chosen by their name or acronym (e.g. "mfd" for the multiple flow direction algorithm) as an alternative to the integer code required by SAGA itself.

3. The RSAGA grid tools are a compilation of additional useful functions for geocomputing and data input/output. This package section implements, for example, generic functions for applying user-defined functions on moving windows ([multi.]focal.function). This allows the user to implement application-specific terrain attributes (such as the wind shelter index for snow distribution modeling) or experiment with new filters.

a) Drawbacks:

-need for frequent file conversions between SAGA GIS and ASCII grid formats

-platform dependence (currently only Windows)

-necessity to synchronize RSAGA with major steps in SAGA GIS development

-provide forward and backward compatibility with SAGA GIS

Raster data Processing in the SAGA GIS is shown in the fig. 2 which explains various features of the region like Forest region, City region, and Agricultural fields. 


\section{ELK Asia Pacific Journals - Special Issue}

ELK

\section{Asia Pacific Journals}

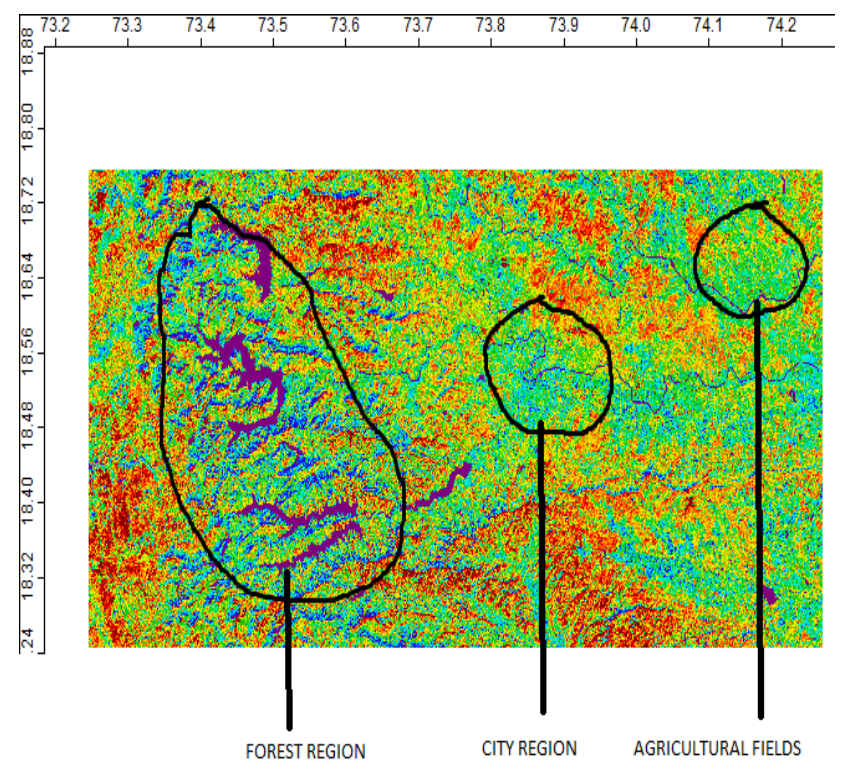

Fig. 2: Map obtained by processing raster data using SAGA GIS

\section{CONCLUSION}

The GIS softwares have proven to be useful in a wide range of fields. SAGA GIS is written in C++ so it will be very power full in computation. The built in modules provide support for several geographic ideas. Good GUI and support for various data formats make it an all-time favourite tool for geoscientists. Execution in the command prompt leads to generation of accurate results. API make way for various languages to be a part of SAGA GIS. As of now, much of the work involving these software is done outside India. It can prove to be of commercial importance to India if there can be a full-fledged implementation. The System whenever implemented in HPC rather than normal system gives better performance in a shorter duration of time.

\section{ACKNOWLEDGMENT}

The author thanks, Dept. of science and technology, Ministry of Science and Technology, Government of India, and the SAGA GIS developer team for creating and maintaining this excellent geo-computing environment, RSAGA GIS Team.

\section{REFERENCES}

[1] Haris H. Khan,Arina, Khan,Shakeel Ahmed, Jerome Perrin" GIS-based impact assessment of land use changes on ground water quality: study from a rapidly urbani zing region of South India”. Springer - Verlag $14^{\text {th }}$ Oct 2010

[2] Olaya V. (2001): A gentle introduction to SAGA GIS. http://www.saga-gis.uni-goettingen.de
[3] Goldi Misra, Nisha Kurkure, Abhishek Das, Shweta Das and Abhinav Gupta, "HPC - A Benediction for Agriculture", International Conference on Information Communication and Management IPCSIT, Singapore, vol.16 (2011).

[4] M.Usha, K.Anitha, Iyappan.L , "Landuse Change Detection through Image Processing and Remote Sensing Approach: A Case Study of Palladam Taluk, Tamil Nadu ", International Journal of Engineering Research and Applications (IJERA) ISSN: 2248-9622, Vol. 2, Issue 4, July-August 2012, pp.289294.

[5] Franco Mantovani, Francisco Javier Gracia, Pietro Domenico de Cosmo, Andrea Suma, "A new approach to landslide geomorphological mapping using the Open Source software in the Olvera area (Cadiz, Spain)" Springer-Verlag 2009.

[6] T.W. Nauman, J.A. Thompson \& N.P. Odgers, Z. Libohova "Digital Soil Assessments and Beyond - Minasny, Malone \& McBratney" ISBN 978-0-415-62155-7.

[7] T. Hengl, G. B. M. Heuvelink, and E. E. van Loon , "Fuzzy disaggregation of conventional soil maps using database knowledge extraction to produce soil property maps On the uncertainty of stream networks derived from elevation data: the error propagation approach," Hydrol. Earth Syst. Sci., 14, 1153$1165,2010$.

[8] J. Böhner, T. Blaschke \& L. Montanarella , "Brenning, A. (2008): Statistical geocomputing combining R and SAGA: The example of landslide susceptibility analysis with generalized additive models.”In: (eds.), SAGA - Seconds Out (= Hamburger Beiträge zur Physischen Geographie und Landschaftsökologie, vol. 19), p. 23-32.

[9] Li Bo, Zhou Zhenliu , Wang Xiangfeng "A Survey of HPC Development", 20International Conference on Computer Science and Electronics Engineering-2012.

[10] Brian E. Mennecke, Martin D. Crossland," Geographic Information Systems: Applications and Research Opportunities for Information Systems Researchers"Proceedings of the 29th Annual Hawaii Intemational Conference on System Sciences 1996.

[11] Bock, M., Böhner, J., Conrad, O., Köthe, R., Ringeler, A. (2007): Methods for creating Functional Soil Databases and applying Digital Soil Mapping with SAGA GIS.

[12] http://www.uni-goettingen.de/de/211052.html

[13] http://www.future-okavango.org/subproject_SP09_2_tfo.php

[14] Institute of Systematic Botany, University Zürich, Biodiversity, Macroecology \& Conservation Biogeography Group, University Göttingen and Physical Geography, University Hamburg.

[15] https://www.geo.uni-hamburg.de/de/geographie.html

[16] http://www.qgis.org/en/site/

[17] http://grass.osgeo.org/ 